
\title{
Besearch S Sulure \\ Comparison of multi-axis printing strategies effects on large-scale 3D printed surface quality
}

\section{Martin Krčma ( $\nabla 152781 @ v u t b r . c z)$}

Brno University of Technology Faculty of Mechanical Engineering: Vysoke uceni technicke v Brne

Fakulta strojniho inzenyrstvi https://orcid.org/0000-0002-5066-7714

David Paloušek

Brno University of Technology Faculty of Mechanical Engineering: Vysoke uceni technicke v Brne Fakulta strojniho inzenyrstvi

\section{Research Article}

Keywords: Robotic fabrication, additive manufacturing, nonplanar 3D printing, multi-axis 3D printing

Posted Date: October 25th, 2021

DOl: https://doi.org/10.21203/rs.3.rs-910728/v1

License: (9) This work is licensed under a Creative Commons Attribution 4.0 International License. Read Full License 


\section{Comparison of multi-axis printing strategies effects on large-scale 3D printed surface quality}

Authors: Martin Krčma, Orcid: 0000-0002-5066-7714; David Paloušek, PhD., Orcid: 0000-0003-46688763

Affiliation: Institute of machine and industrial design, Faculty of mechanical engineering, Brno university of technology, Czechia

Corresponding author: Martin Krčma, martin.krcma1@vut.cz

Keywords: Robotic fabrication, additive manufacturing, nonplanar 3D printing, multi-axis 3D printing

\section{Abstract}

The purpose of this study is to determine impact of several multi-axis 3D printing strategies on buildability, surface quality, accuracy and strength of large scale single-walled object (printed in a so called vase mode). To achieve this goal, test objects were printed using four distinct printing strategies by an industrial robotic arm and a pellet-fed screw extruder. The strategies tested in this study are: regular 3-axis deposition with planar layers, 5-axis deposition with planar layers, 3-axis deposition with non-planar layers, and 5-axis deposition with non-planar layers. Custom scripts for nonplanar slicing and for tilt control during multi-axis printing were developed to achieve these prints and are explained in this study. The results were evaluated using 3D scanning and mechanical testing, and surface accuracy, surface roughness and layer adhesion strength were compared. The most important findings are: 1 . 5-axis motion alone does not improve the results of the printing, 2. While nonplanar printing can improve surface quality its usability is geometry dependent, 3. Multi-axis nonplanar printing, even with partial tilt $\left(30^{\circ}\right)$ can expand printability with enhanced quality to at least $75^{\circ}$ overhang angle. Future potential of these methods and requirements to achieve them is discussed.

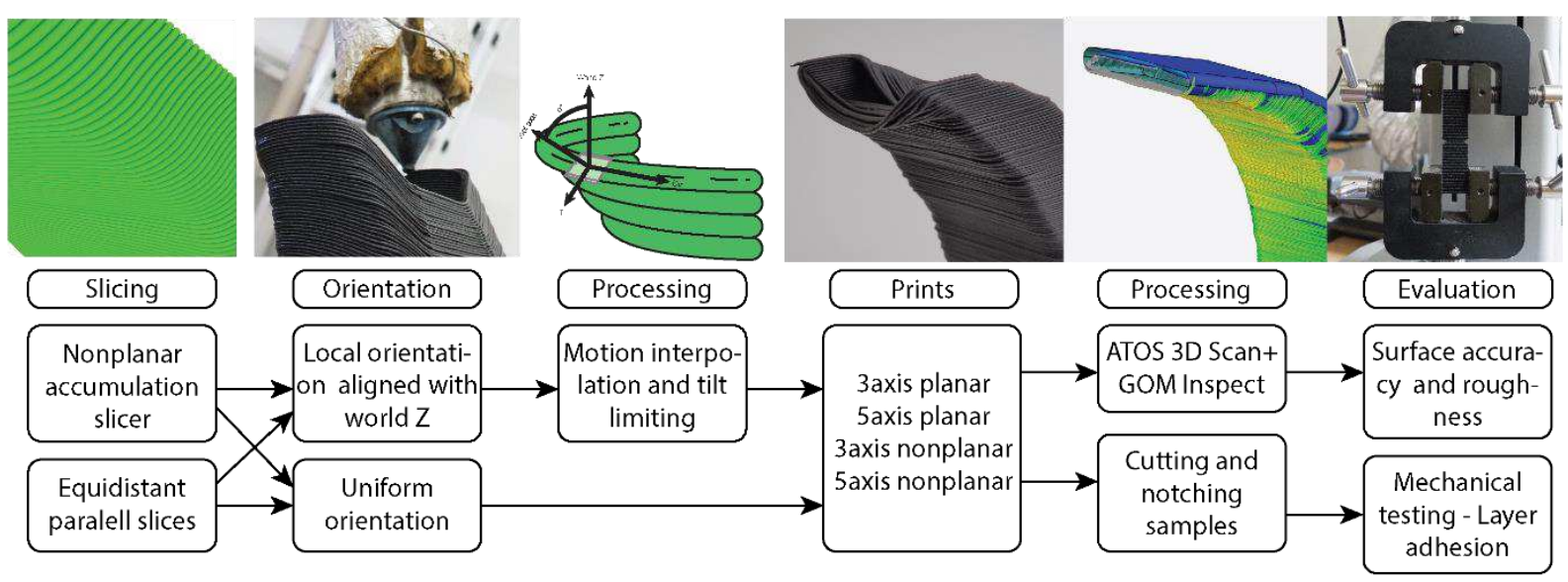

Fig. 1 Graphical abstract

\section{Introduction}

The limitations of layer by layer processes, such as FFF (Fused Filament Fabrication) are well known to its users and partially result from the slicing process that uses equally spaced parallel layers. This causes anisotropy and reduction of surface quality due to stair-stepping [1], both of these dependent on build orientation [2]. Lately, a wide array of publications pushing these limitations has been coming out, in areas of multi-axis printing, support-less printing and non-planar printing strategies. One possible approach is the deconstruction of the model into parts with different slicing directions based on local geometry [3], [4]. The second study is closely related to another area of research, and that is nonplanar, or curved layer printing. Current publications are mainly focused on the data preparation and path 
planning aspects of the processes. The presented study aims to analyse the impact of these strategies on the resulting objects more closely and discuss usability of these strategies for expanding the application boundary of the layer-by-layer extrusion printing methods. Other areas where multi-axis printing was used are DED using robotic arm [6] or UV resin based accumulation processes. [7]

The focus is on usability and evaluation of these methods for large scale printing, because this area could benefit more from reducing the overall amount of material used and the need for support structures than desktop scale machinery. [5] Large scale prints are commonly only single-walled and contact area between subsequent layer has much bigger impact on print quality, surface roughness due to stair stepping is significantly more noticeable for large layer heights, as are the errors that retractions bring into prints.

The main difficulty in non-planar printing lies in the multi-faceted problem of slicing/path planning for such a method. Universal slicing algorithms have only recently been presented by researchers and utilize computationally difficult and user inaccessible voxel accumulation based or iso-parametric/ gradient based methods. [8],[9] Previous, more accessible approaches used transformation based methods, often utilizing a constant slicing geometry, either an arbitrary one or one extracted from the part, for the entirety of the build. These methods were suitable only for simple geometries and often had to make significant sacrifices in surface quality.

\section{State of the Art}

The improvement to buildability that can be gained from varying the build orientation was demonstrated by Zhao et al. utilizing incline layer slicing [3] or by Kubalak et al. utilizing a multi axis system. [10] Earliest mention of a technique to print curved layers was presented under the name CLFDM, with main benefits to printing near-flat thin walled parts such as turbine blades. [11] Approachable methods to generate curved print paths by cylindrical transformation are described by Zhao et al. [12]

Schuh et al. described the problem with varying layer heights and stair-stepping on angled surfaces and demonstrated a method to eliminate it utilizing printing along local surface orientation with adaptive layer heights. [13] Limitations of layer stacking and nozzle reach for volumetric print paths, as well as multiple methods to generate such paths are described in B. Ezair et al. [14]

The method to print curved top surfaces on a 3-axis delta-type robot was presented by R. Allen and R. Trask. [15] Slope angle of the computed slices is a limitation, especially for 3-axis printers, and has to be controlled to avoid collision. An algorithm that can decompose whole objects for printing on a 3axis machines was described by CurviSlicer [16]. The limitation can be overcome by using multi-axis motion to reorient the build orientation, either by dividing the model into parts that can be printed with parallel layers [17], [10] or by using local tangent direction and nonplanar trajectories. A method for computing these trajectories was described by $\mathrm{Xu}$ and collective. [18]

Fang et al. presented an algorithm for nonplanar volumetric slicing and showed that printing nonplanar layer oriented along stress lines can increase strength of prints by more than 6x. [19] C. Dai et al. describes voxel based nonplanar slicing algorithm for support less printing, that ensures printability for multi-axis printing by generating convex shells. [20]

\section{Methods}

To evaluate usability of the different methods a test object was designed, and 4 different print strategies were prepared, manufactured and evaluated. The strategies are: 3-axis deposition with planar layers, 5-axis deposition with planar layers, 3-axis deposition with non-planar layers, and 5-axis 
deposition with non-planar layers. The 5-axis deposition methods are intended to improve surface quality by orienting the extruder nozzle along local surface tangent. The nonplanar strategy focuses on keeping actual distance between layers, measured along surface orientation, constant for the entire build.

\subsection{Samples and trajectories}
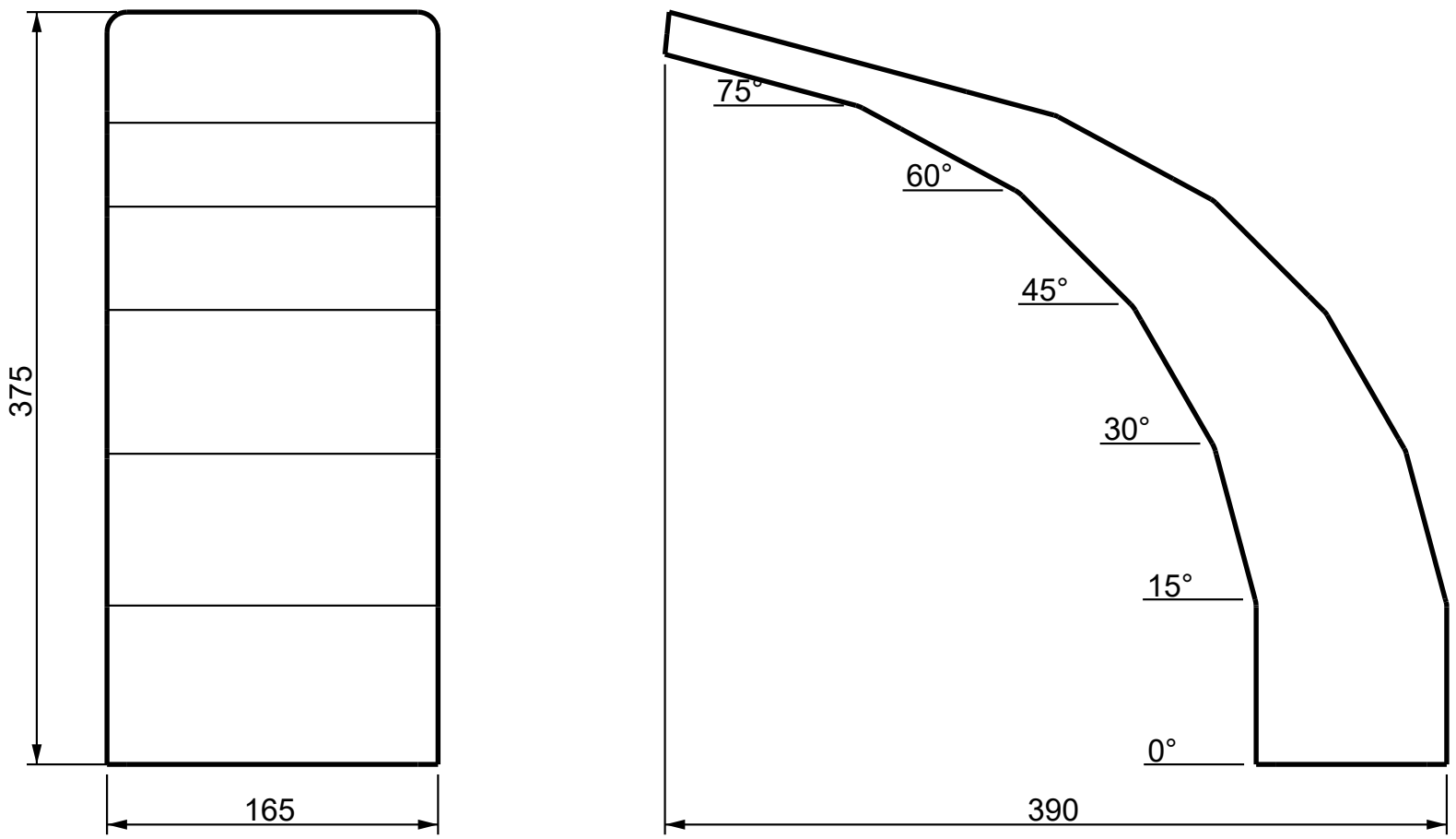

Fig. 2 The dimensions of the test tower

The test object used for the comparison is an overhang tower, stepped by $15^{\circ}$ from $0^{\circ}$ to $75^{\circ}$. The tower dimensions are in the Fig. 2. The tower is designed in such a way that the trajectories are arrayed in straight, parallel layers on the inside overhang surfaces for all towers and these surfaces are then used for analysis and comparison. Tower cross-section is constant to keep layer printing times for 3 axis methods constant as well. They vary slightly for nonplanar methods, (the nonplanar trajectory is $6,4 \%$ longer at the $60^{\circ}$ segment, as the nonplanar trajectory covers the same area with more layers) but not enough to warrant designing a different test object.

The test objects were modelled, sliced and resulting robot motions programmed in Rhinoceros 3D and Grasshopper environment, with kuka.prc [21] plugin being used for robot code generation. Custom algorithms were developed for slicing and motion planning in the Grasshopper environment.

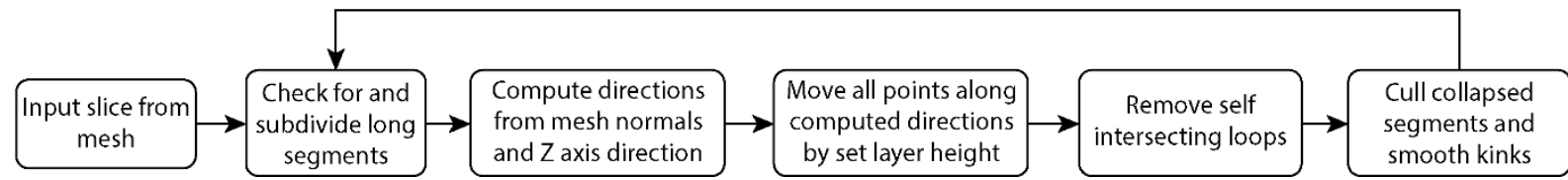

Fig. 3 Scheme of the nonplanar slicing algorithm 
The nonplanar slicing algorithm works iteratively, calculating the position of the next layer from the last to keep the actual layer height the same as the nominal layer height for all points. The basic workflow can be seen in Fig. 3. Fig. 4 shows both used trajectories, and Fig. 5 displays deviation from nominal layer height for both approaches, with points with spacing of $<2 \mathrm{~mm}$ coloured in green and $>2 \mathrm{~mm}$ coloured in red. The range of distances is 2 to $7.72 \mathrm{~mm}$ for the planar trajectories and 0.7 to 4.1 for the nonplanar approach. Actual layer height, sorted from lowest to highest is also displayed on charts as a part of Fig. 5. Layer height variation in the planar trajectories is easily explained by the overhang angle, as the Actual layer height = nominal layer height $/ \cos \left(\right.$ overhang $\left.^{\circ}\right)$. Any significant layer height deviations in the nonplanar trajectories come from slicing artifacts around sharp corners and these are rare outliers, as can been seen in the point distribution chart.

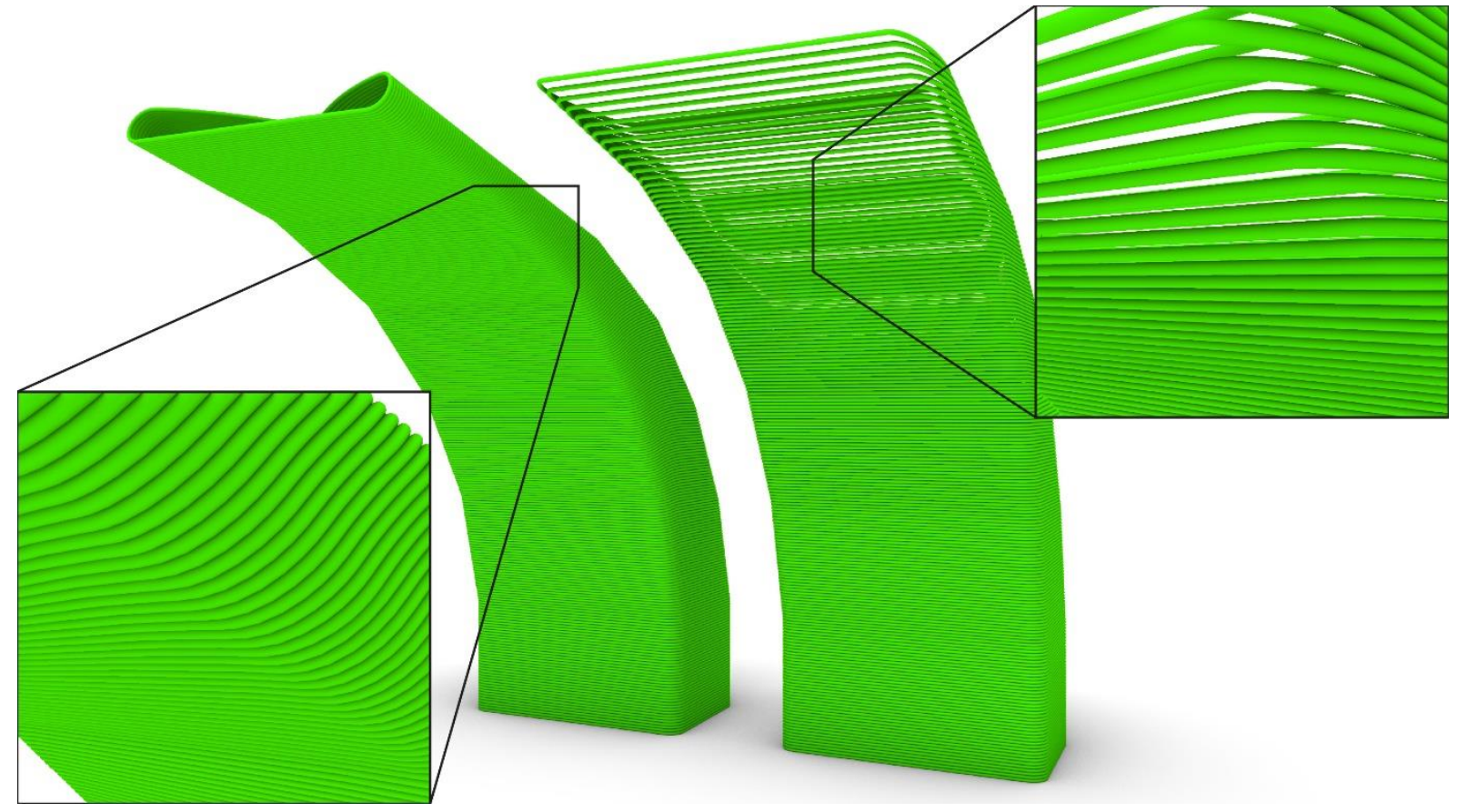

Fig. 4 Comparison of the two used trajectories

The planar trajectory is $88227 \mathrm{~mm}$ long (est. print time $4 \mathrm{~h} 55 \mathrm{~m}$ ) and the nonplanar trajectory, without the top extension needed to avoid open loops and thus covering the same surface area, is $113711 \mathrm{~mm}$ $(6 \mathrm{~h} 15 \mathrm{~m})$. Both trajectories were printed using both the regular, 3-axis printing and the 5 -axis printing, where the extruder is oriented along the local surface tangent and perpendicular to the trajectory. The maximum allowed angular deviation from the world $Z$ axis is set to $30^{\circ}$, mainly to ensure proper gravity feeding of the granulate from the hopper and to increase the usable build envelope. This limit is enforced by the tilt control script, explained in the next section.

The motion planning is carried out followingly: First, the orientation frames are calculated for each point as a cross product of the direction of the trajectory as the first vector and the local normal as the second vector (Fig. 6). Then, simple interpolation is applied by averaging each points orientation with 
its closest neighbours to ensure smooth robot motion even in sharp corners. Before calculating final set of robot frames, tilt control is performed.

1. Non-planar trajectory

\section{$2.0 \mathrm{~mm}$}
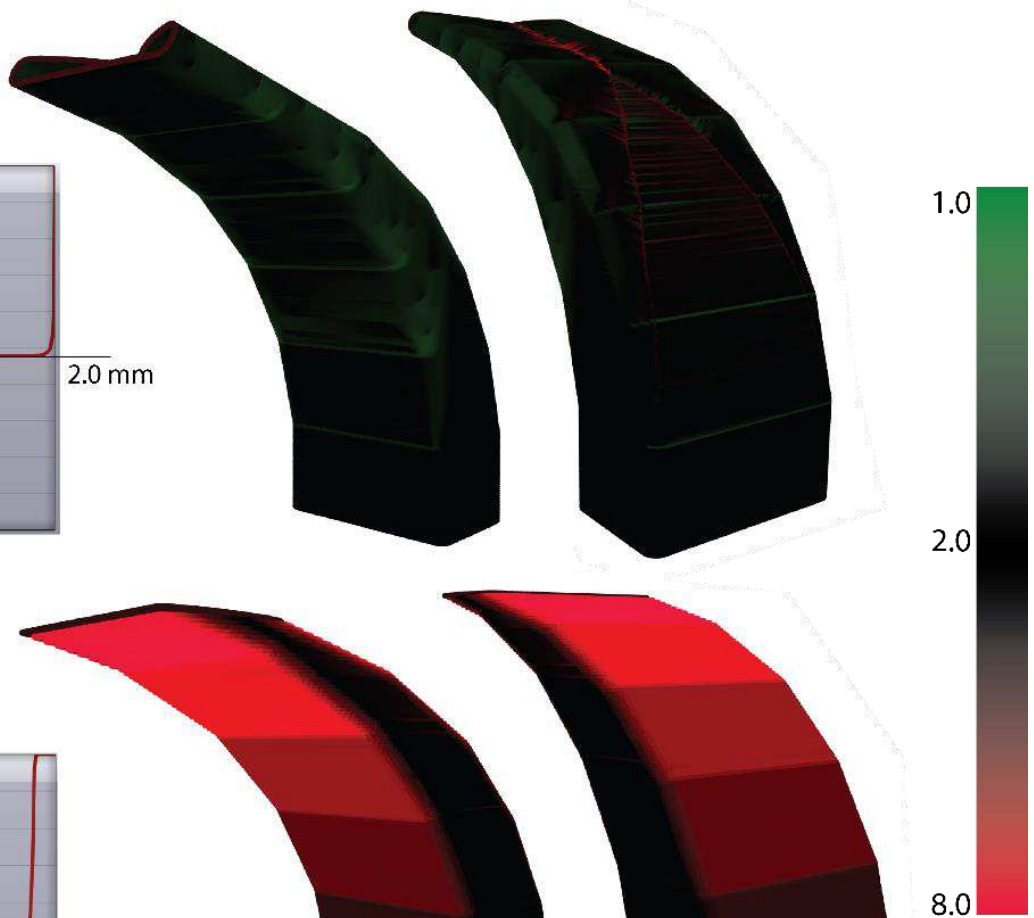

2. Common slicing
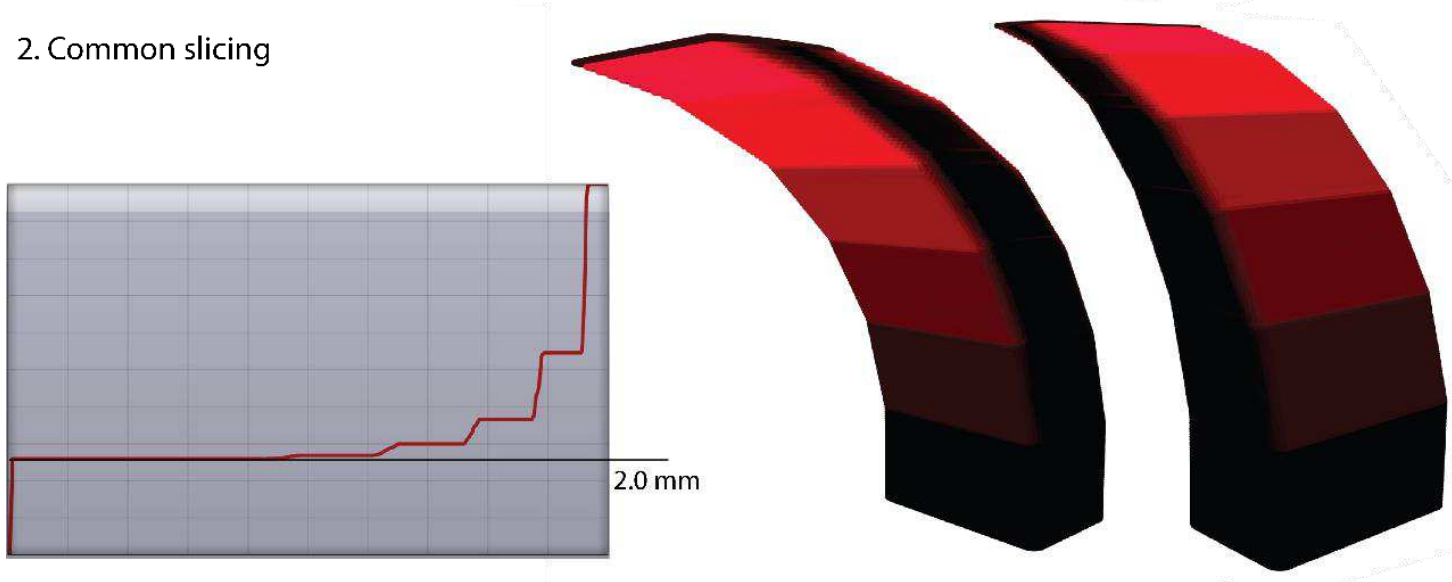

8.0

Fig. 5 Visualisation of the deviation from layer height for both trajectories using a plot of distances between all adjacent points and coloured visualisation on the mesh

The tilt control script works by evaluating the angle ( $\alpha^{\circ}$ in Fig. 6 ) between the intended orientation of the extruder and the world $Z$ direction and checking it against the threshold. If the angle $\alpha$ exceeds the threshold, the frame is replaced by one on an identical plane (described by the World $Z$ vector and the surface normal) with the deviation angle set at the threshold.

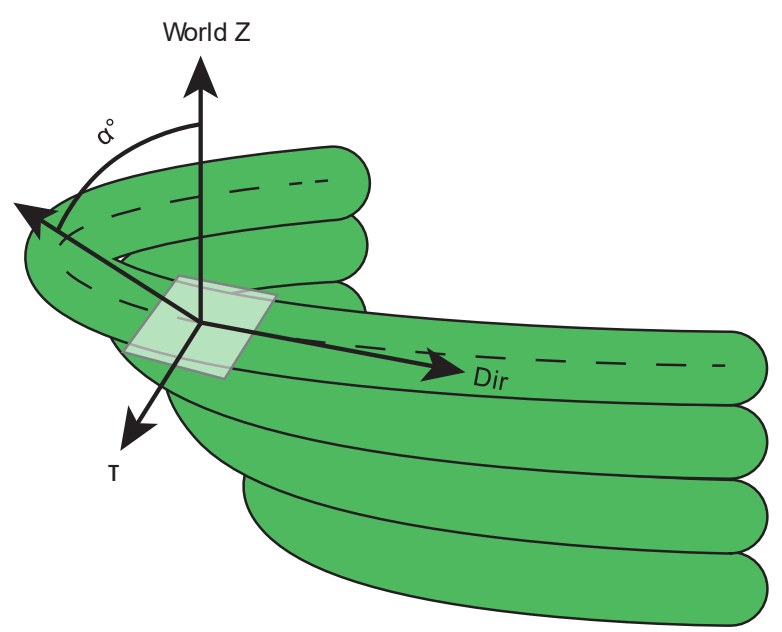

Fig. 6 Tool orientation for 5-axis trajectories 


\subsection{Materials}

The robotic arm used as an 3d printer gantry was Kuka KR $60 \mathrm{HA}$, with KRC 2 controller. The extruder is a custom-built single screw extruder with a diameter of $30 \mathrm{~mm}$. Nozzle diameter is $5 \mathrm{~mm}$, with an extended tip in order to increase clearance when printing a non-planar paths. The end point speed was reduced to $5 \mathrm{~mm} / \mathrm{s}$ to allow sufficient cooling of the deposited layer before another one is deposited [22]. The assembly can be seen in Figure 7.

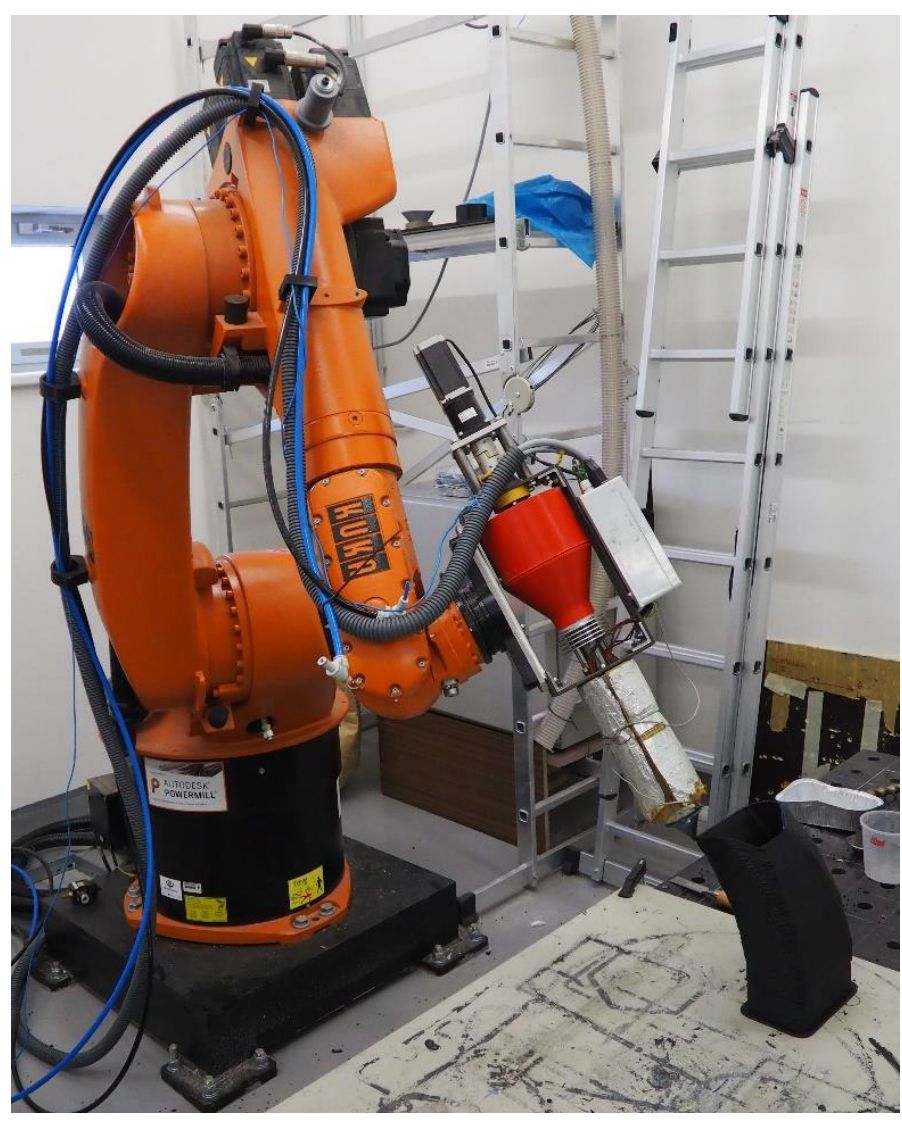

Fig. 7 Robotic arm with the screw extruder during a multi-axis print

The test objects were printed using Remark Plast Remapylen GF 30 (rPP GF 30). This material is a widely available engineering plastic, that promises economical printing and is very resistant to degradation in the melt extrusion. The semi crystalline nature of the polymer makes it prone to warping during the printing process, this behaviour is reduced by the high fibre filler percentage. [23] The extruder temperature was set at $230^{\circ} \mathrm{C}$, the layer height at $2 \mathrm{~mm}$.

The resulting specimen were then 3D scanned using ATOS Triple scan optical scanner. 3D optical digitization is based on active fringe projection and triangulation.

Process of 3D data digitization:

1. Surface matting with chalk spray

2. Reference points placement on the part surfaces

3. 3D digitizing with a rotation table

4. Post-processing and evaluation of deformations

The measurements were performed with the scanner setup according to "VDI/VDE 2634, Part 3 Optical 3D-measuring systems, multiple view systems based on area scanning". The measurements in this study were done using MV560 lenses $(560 \times 420 \times 420 \mathrm{~mm})$ calibrated in a standard arrangement. The 
measuring point distance is $0.176 \mathrm{~mm}$, i.e. 6 points $/ \mathrm{mm}$, the recommended reference points diameter is $3 \mathrm{~mm}$, measuring distance $830 \mathrm{~mm}$, camera angle $27^{\circ}$, focal length of camera lenses $24 \mathrm{~mm}$ and focal length of projector lens $30 \mathrm{~mm}$.

CAD alignment (Fig. 8) was based on fitting planes in the $0^{\circ}$ overhang sections of the printed objects and aligning them with the original CAD surfaces. This method was chosen instead of best-fit to properly represent the progressive deviation that occurred during the prints. The point cloud reconstruction, alignment to the nominal CAD model and deviation analyses were performed in GOM Inspect.

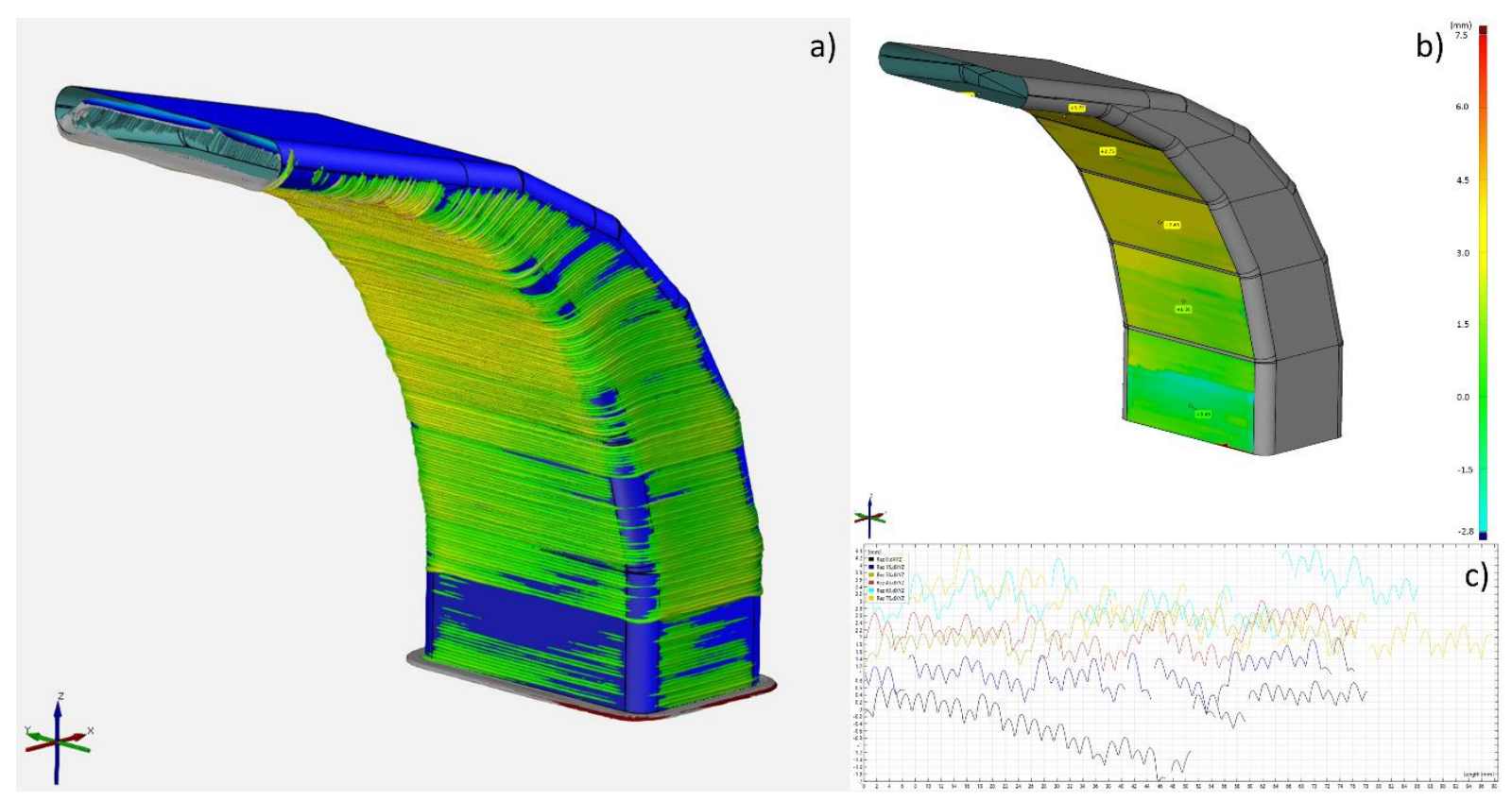

Fig. 8 a) 3D scanned mesh reconstruction of the 5axis nonplanar test object aligned with the nominal CAD data b) The surfaces evaluated for deviation analysis c) A set of profile curves exported from GOM Inspect

On the resulting meshes, mean surface deviation analysis and surface profile extraction for surface roughness ( $\mathrm{Ra}$ - arithmetic average height) calculation were performed. For the surface deviation analysis, the mean absolute deviation from the nominal CAD model is presented. Evaluated surfaces can be seen in Fig. 8, b). For the surface roughness, profile curves were extracted, and $R a$ was calculated using the Formula 1, which is the digital version of the Ra expression. The profile curve was filtered using sliding average with a wide window to eliminate influence of possible surface defects such as waviness. [6] After this, the mean $m$ and resulting $R a$ were calculated.

$$
R a=\left(\left|Z_{1}-m\right|+\left|Z_{2}-m\right|+\cdots+\left|Z_{N}-m\right|\right) / N
$$

\subsection{Layer adhesion testing}

Slabs were cut from each tier of the overhang towers on the internal wall where the layers run in parallel for both slicing approaches. Layer adhesion samples were then cut and ground from these slabs, dimensions $100 \times 20 \times 5$, notched in the middle along a layer boundary to ensure delamination in the preferred position. The specimen can be seen in Fig. 9. The V-shaped notch was broached, $4 \mathrm{~mm}$ in depth, $60^{\circ}$ tip angle. Specimens were kept sealed with a desiccant for 14 days. 


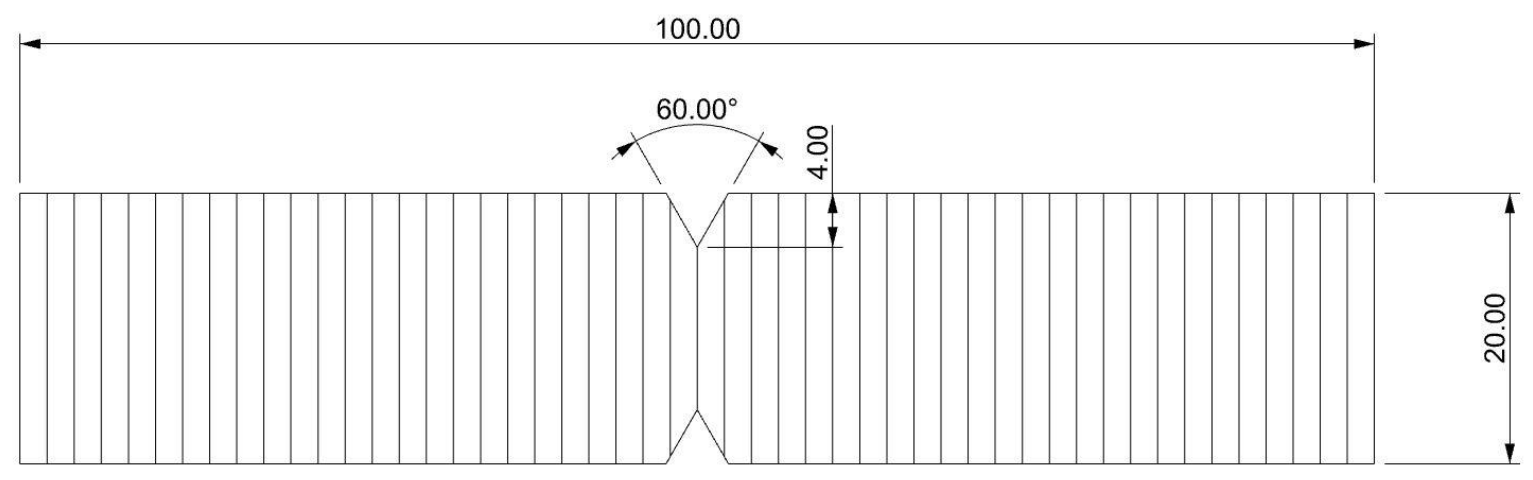

Fig. 9 The layer adhesion sample dimensions

The samples were then tested for layer adhesion on an Imada MX2 test stand, equipped with a 2,5 kN load cell, with the loading speed was $10.0 \mathrm{~mm} / \mathrm{min}$. Maximum force was recorded, and the fracture surface was measured. A test sample loaded in parallel jaws can be seen in Fig. 10.

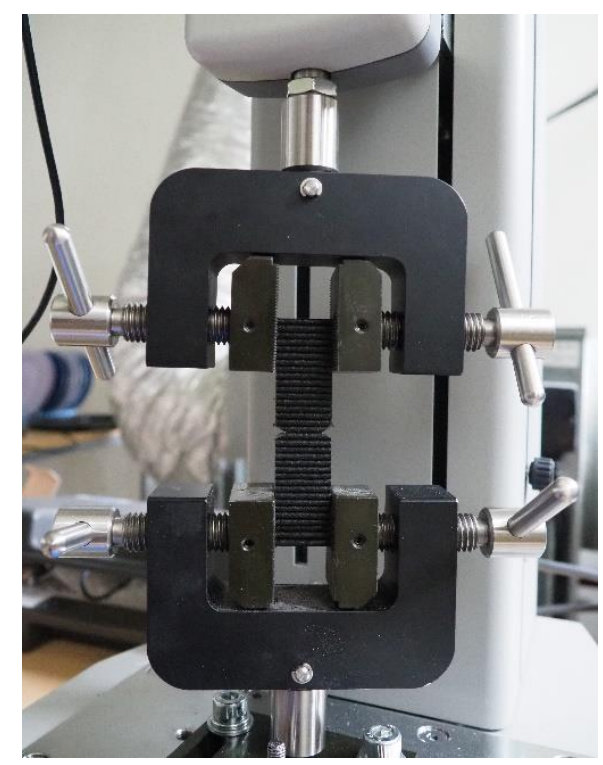

Fig. 10 A layer adhesion specimen loaded in the testing machine

\section{Results}

\subsection{Printing results}

\begin{tabular}{|l|l|l|l|l|l|l|}
\multicolumn{1}{l}{} & $0^{\circ}$ & $15^{\circ}$ & $30^{\circ}$ & $45^{\circ}$ & $60^{\circ}$ & $75^{\circ}$ \\
\hline 3-axis planar & ok & ok & ok & ok & fail & fail \\
\hline 5-axis planar & ok & ok & ok & ok & fail & fail \\
\hline 3-axis nonplanar & ok & ok & ok & fail & fail & fail \\
\hline 5-axis nonplanar & ok & ok & ok & ok & ok & ok \\
\hline
\end{tabular}

Table 1 Overview of printing results by overhang angle

Of the four builds, the only one to finish printing was the 5-axis, nonplanar build (Table 1). All the builds before testing can be seen in Fig. 11. Both the planar builds failed after $45^{\circ}$ of overhang by failure to stack subsequent layers. The 3 -axis nonplanar test failed after $30^{\circ}$ when the nozzle contacted the previous layer, due to slope becoming too steeply angled. The build appeared to continue, but it had 
separated from the build platform and shifted. This has caused the segments after the $30^{\circ}$ overhang to become printed out of alignment with the underlying geometry.

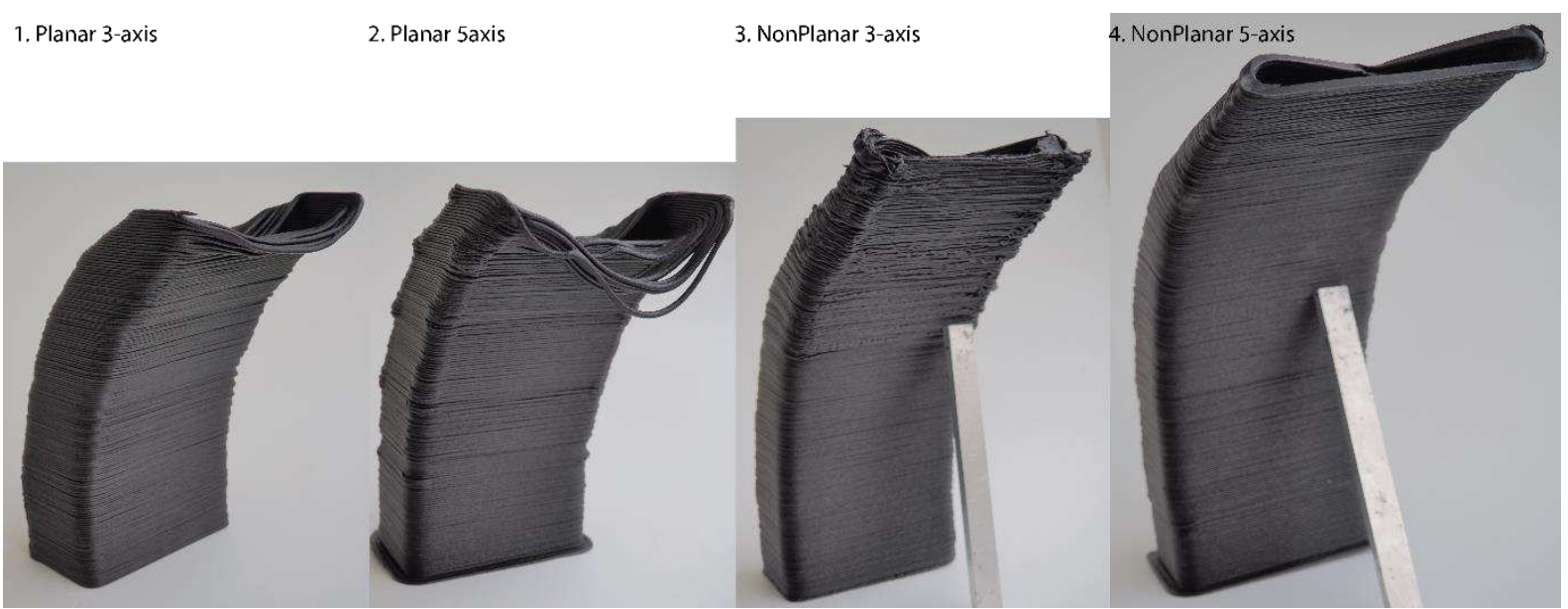

Fig. 11 All the prints side by side, from left to right: Planar 3-axis, Planar 5-axis, Nonplanar 3-axis, Nonplanar 5-axis

Notable issues recorded during printing: Feeding issues, causing under extruded segments in the 5axis builds. The under extruded areas were excluded from all analyses. Both the 5-axis samples had over extruded areas in the corners of the transitions between overhang angles. This is due to a slicing error, the double curved surface patches generated large number of points, causing the robotic arm to slow down. The last, $75^{\circ}$ overhang segment of the nonplanar 5 -axis is under extruded, as the nozzle was near contacting the previous segment and reaching failure state.
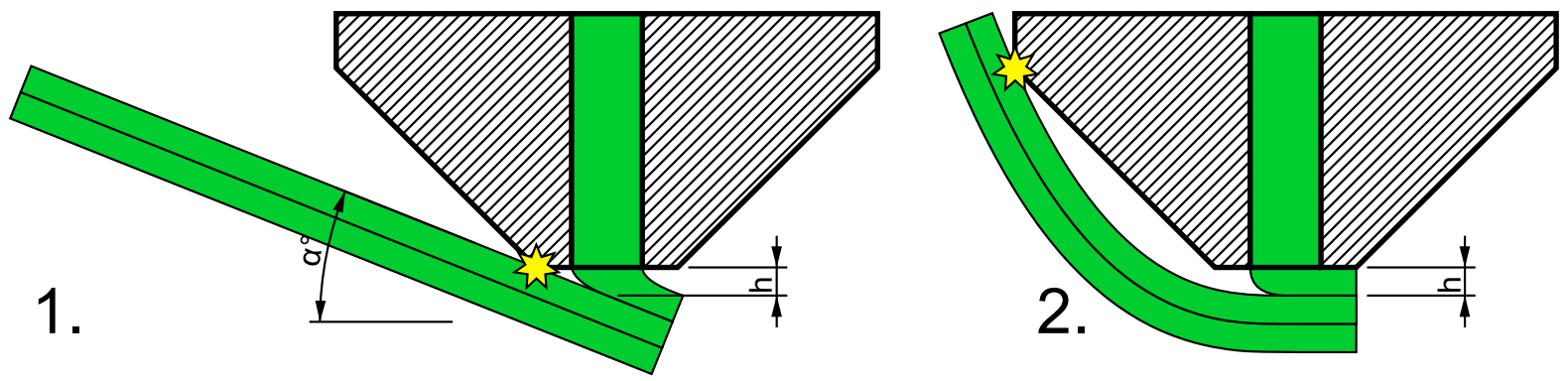

Fig. 12 1. Collision due to the slope steepness 2. Collision with surrounding geometry

The results show that using multi-axis printing with conventional slicing technique does not improve printability on its own, as well as showcasing the problems that arise with the use of nonplanar trajectories. The nonplanar 3-axis method is especially limited and failed because of slope angle limitations. The nonplanar 5 axis method has a much wider area of applicability, but also increased requirements due to possibility of collision. Geometric limitations are displayed in picture Figure 12. Figure 13. shows near collision due to local geometric limitation in the 5 axis test object. 


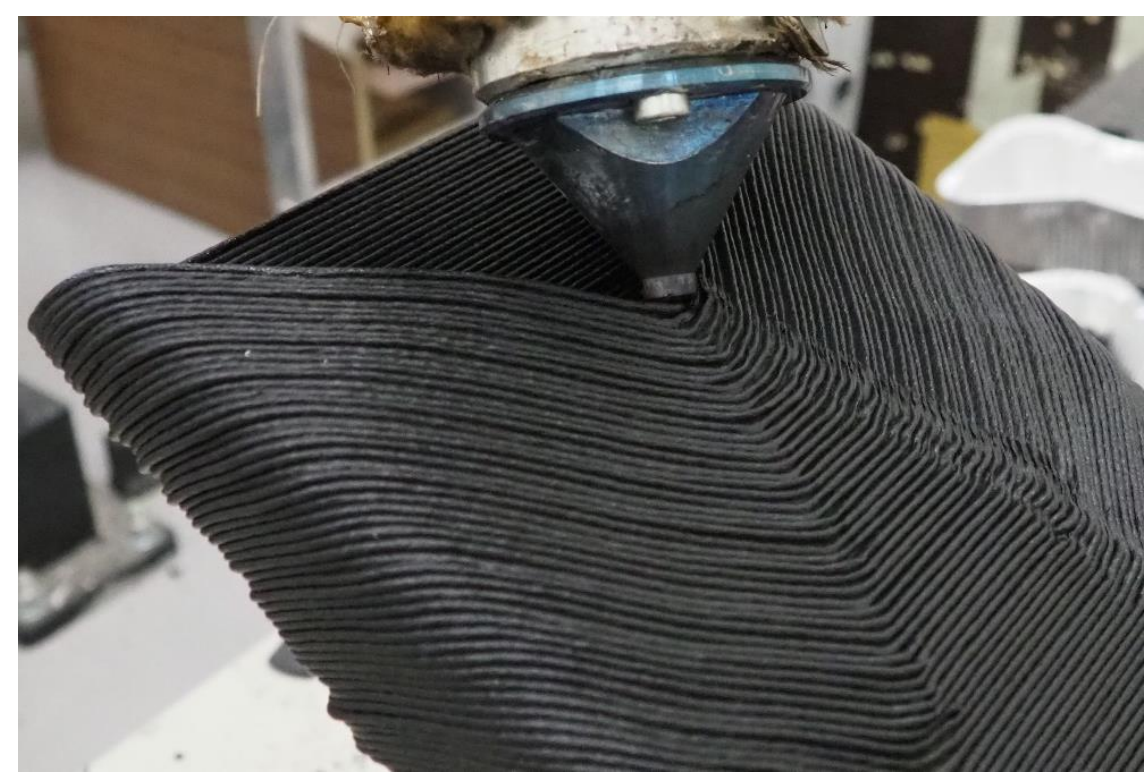

Fig. 13 Nozzle near contacting local geometry during the $75^{\circ}$ segment

\subsection{Accuracy}

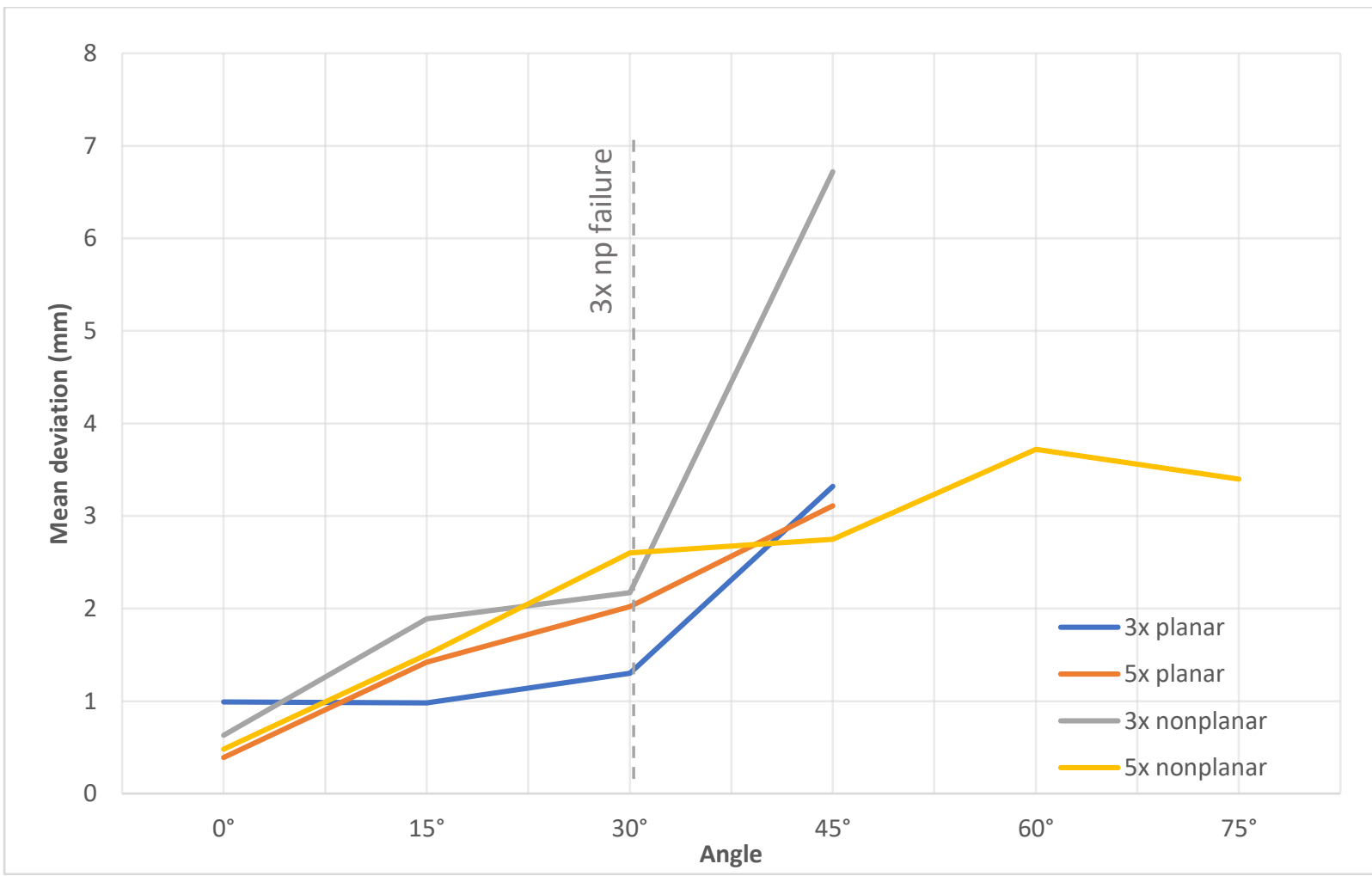

Figure 14 Mean deviation chart 


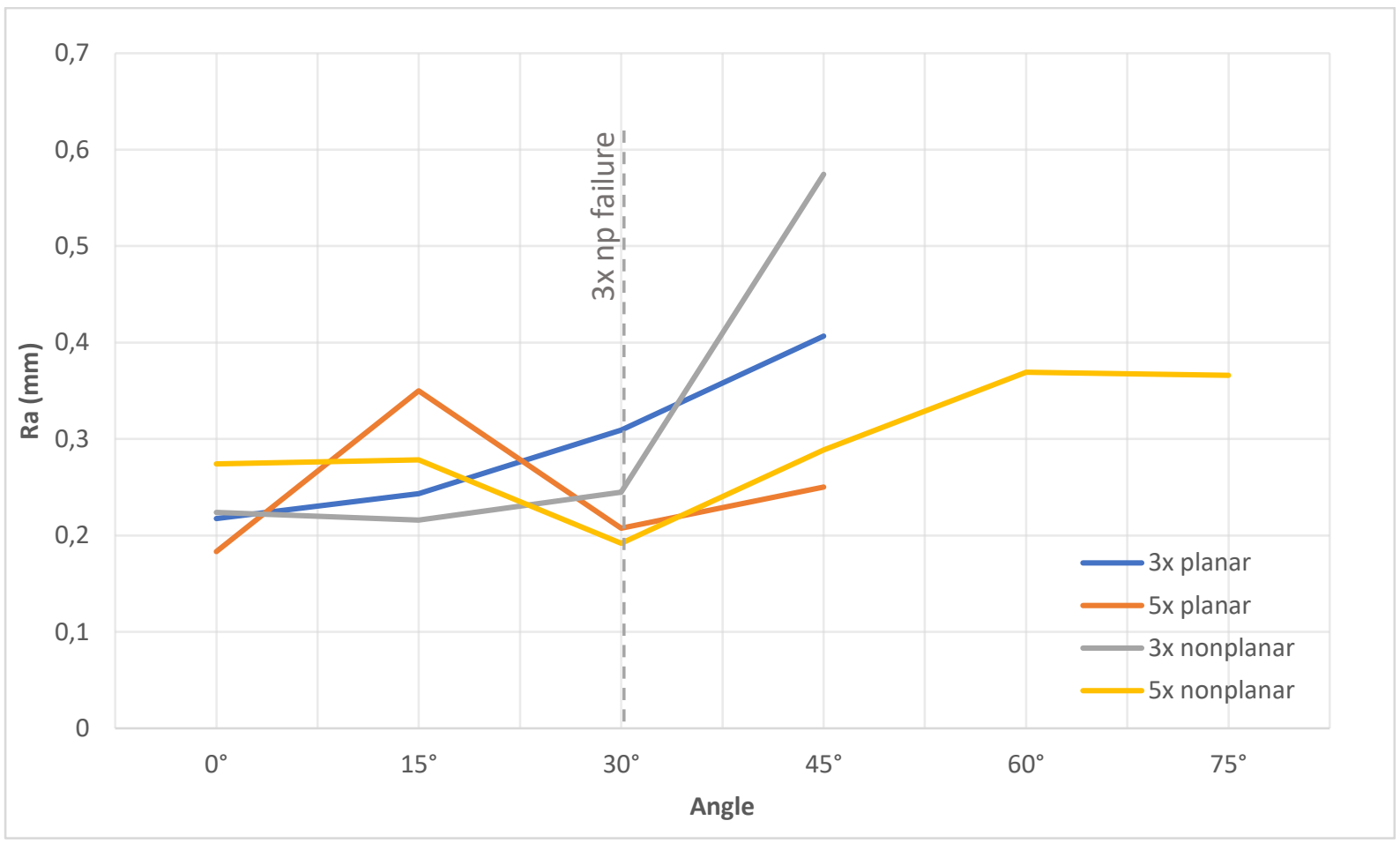

Fig. 15 Surface roughness chart

The results of comparisons between the four test objects can be seen in Fig. 14-16. The 3-axis nonplanar test object is evaluated even beyond the point of print failure when possible, otherwise it is excluded.

The Fig. 14 shows the total mean deviation, including the deviations caused by geometric deviation, surface roughness and the deviations caused by technology - such as differences in print path width. Initial deviation, caused by thickness of the print path lies between $0,4-1,0 \mathrm{~mm}$. The results show consistent increase for all methods. The deviation increases for each method until its respective point of failure, with the deviation of the last correctly printed segment lying between 2-4 mm for all methods.

Surface roughness can be seen in Fig. 15. After the first segment, the surface roughness starts to increase for the 3-axis planar method starting at 0,22 $\mathrm{mm}$ and reaching $0,41 \mathrm{~mm}$. The 5 axis and nonplanar methods, while sometimes worse at low overhang angles, manage to keep lower surface roughness at hight overhangs until the tilt control allowance runs out. Surface roughness is the only area where the 5-axis planar strategy appears to bring benefits, as the print finished with nearly the same roughness as at the start. 


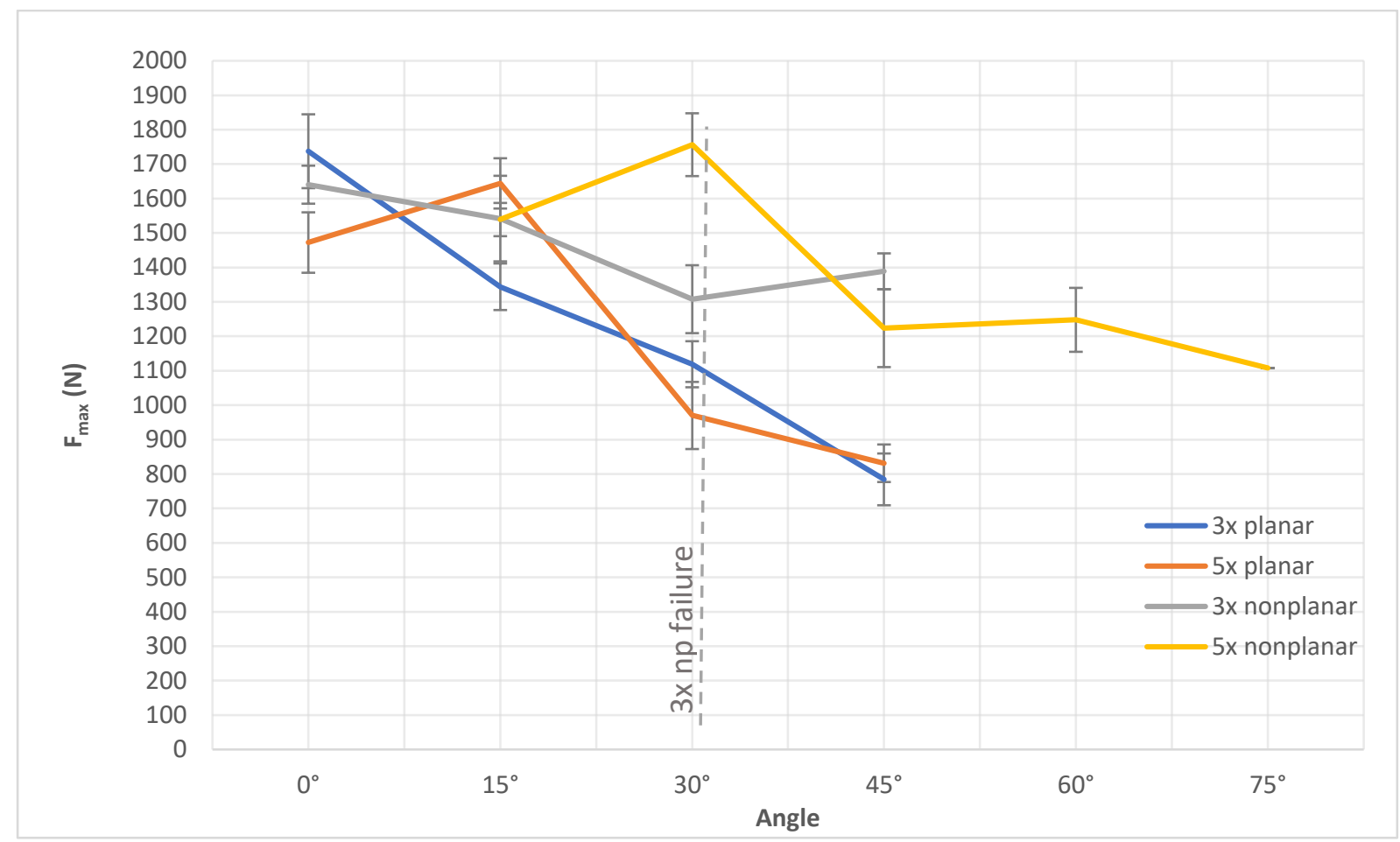

Fig. 16 Maximum delamination force chart

\subsection{Mechanical testing}

Maximum average delamination force $\left(F_{\max }\right)$ can be seen in Fig. 16. Slight initial differences between the test objects could be due to the slicing errors, but the percentual differences between different overhang angles of the same test object can still be evaluated. The planar slicing methods showed rapid decline in the delamination force (from $1737,4 \mathrm{~N}$ to $784,5 \mathrm{~N}$ ), which can be explained by the decrease in contact area between adjacent print paths. The 5 -axis planar test lost about $46 \% \mathrm{~F}_{\max }$ and did not show significant improvement over the 3 -axis test, which lost about $54 \% F_{\max }$.

For the nonplanar tests, some decrease in $\mathrm{F}_{\max }$ was present, but to a much lesser degree. The 3-axis nonplanar test object lost $25 \% \mathrm{~F}_{\max }$ at the $45^{\circ}$ overhang segment, while the 5 -axis lost $20 \%$ and $30 \%$ at the $75^{\circ}$ segment. The decrease in delamination force is much more gradual for nonplanar printing methods. These methods could be promising in preserving strength in vertical direction of prints, which is the direction most susceptible to anisotropy in FFF and similar methods. [2] This anisotropy is even more prominent in fibre filled materials such as the PP GF 30 used in this study. [24] The method would also bring consistency into printed parts by decreasing the dependency of mechanical properties on the overhang angle.

\section{Discussion}

This article explores the impact of individual multi-axis printing methods. Slicing for such methods must deal with the problems of geometry collision, either by slope steepness or by local geometry (Fig. 12). For the process to be reliable, either the slicer has to generate trajectories with the geometric constraints included in the slicing, or each layer has to be collision checked. A possible approach to generate guaranteed collision free trajectories could be separating areas of the layer into multiple levels, based on the local steepness, similar to CAM finishing strategies for shallow areas. Another approach to generating layers with guaranteed printability is generating "convex hull layers" [20]. 

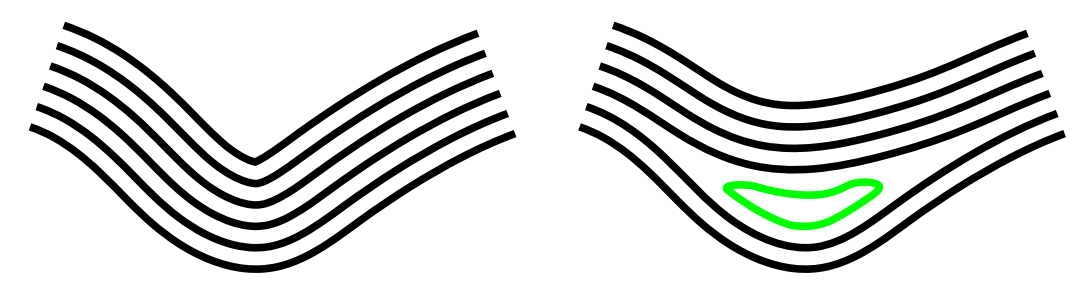

Fig. 17 Suggested technique for sharp corner prevention by partial layer insertion

The slicing algorithm used in this study is incomplete. It is only suitable for slicing vase mode objects with a single continuous perimeter. It also does not include any pre-calculation to ensure that the calculated trajectories cover the entire object. If the sliced object has areas with much greater geodesic surface distance than others it is possible that the algorithm will fail or will not cover the entire surface. This can be overcome by calculating layers with variable height. The nonplanar trajectories used in the study did not cover the entire test tower. The upper area of the tower was excluded to avoid printing partial perimeters with retraction. Due to its ability to cover horizontal areas and even negative overhangs, the slicer can be still used for a wide variety of geometry. Computational intensity of the presented non-planar slicing algorithm is higher than that of a constant layer height slicing. It is influenced to a high degree by density of the input mesh and user set parameters, mainly the max. length of a segment before point insertion. Regular slicing of the test object takes roughly 6 seconds, while the runtime of the non-planar slicer is about 90 seconds on an i7 7700 equipped machine. Demonstration of the slicer presented on a sample geometry can be seen in Fig. 18.

a)

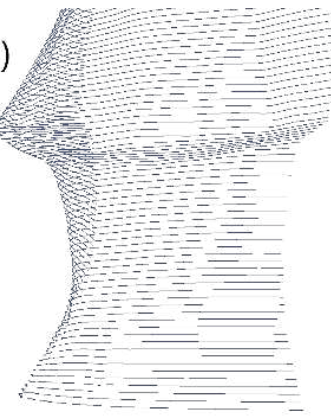

b)

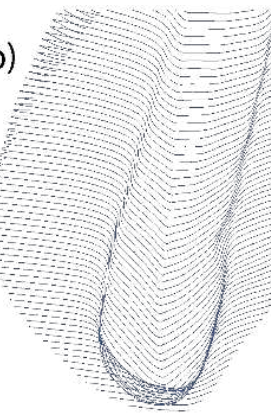

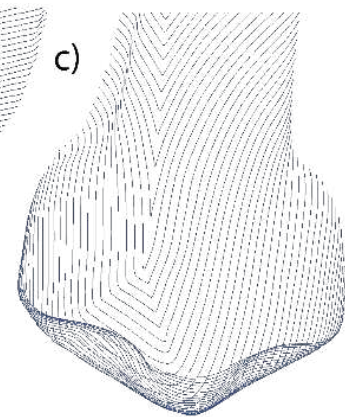

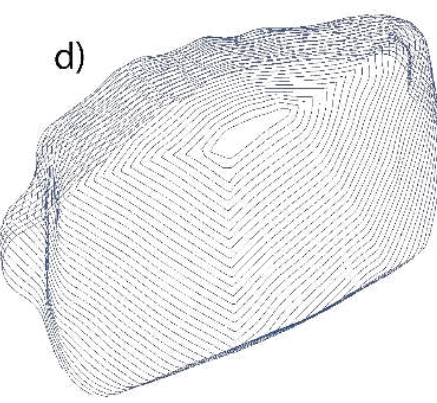

Fig. 18 Demonstration of non planar slicer used on an organic mesh a.b) correctly arrayed trajectories around narrow parts and overhangs c) tight corner with trimmed loops d) failure to cover tight area at the end of the print

Important part of the nonplanar slicing script is the clean-up after a layer shift. The most problems arise in areas that have significantly smaller curvature radius than layer height and after rapid decrease in cross section area. These areas can produce self-intersecting loops in the trajectory. These have to 
be detected and eliminated, shown in Fig. 19, or precautions have to be taken, such as preventive filleting of detected sharp corners.
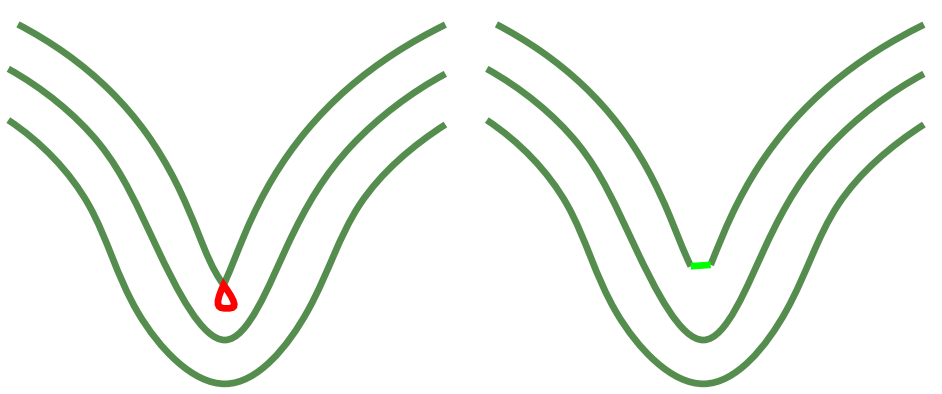

Fig. 19 Loop creation on sharp corners and prevention by filleting

The main reason for using this transformation based method instead of isocurve/isoparametric based one is the ability to calculate trajectories for multi-surface or mesh objects and the ability to generate continuous curves for vase mode printing of single walled objects, without the need to check surface coverage and insert additional curve segments as in older methods [25] and with the need for supports mostly eliminated.

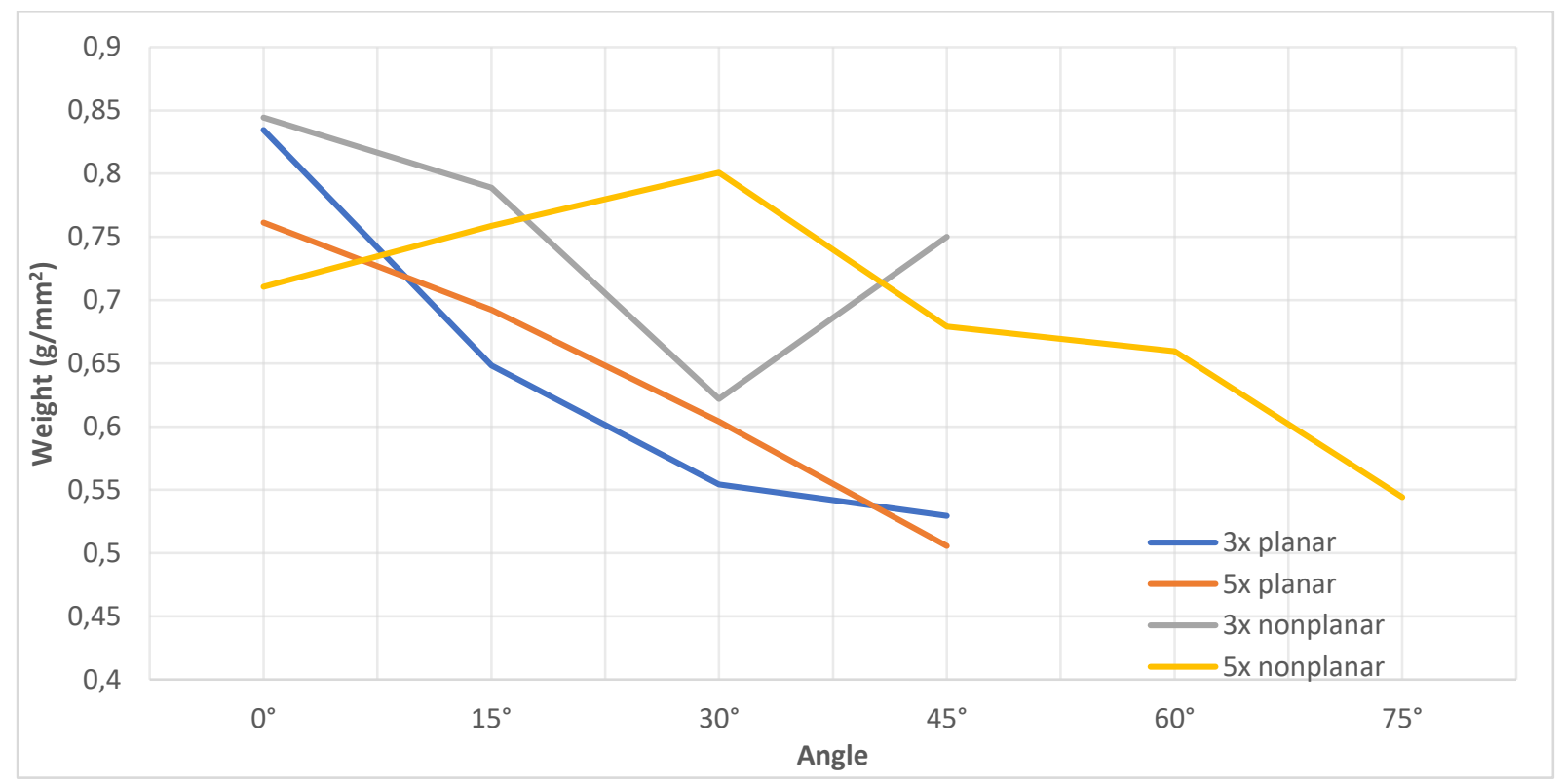

Fig. 20 Area density of the delamination samples

The improved results of mechanical testing should mostly be attributed to the nonplanar trajectories keeping the amount of material deposited nearly constant independent on the overhang angle. Area density of the samples (Shown on Fig. 20) partially supports this claim. The influence of different layer orientation in regard to tensile stress between the 3-axis and the 5-axis strategy is harder to establish, but it does exist. Literature suggests that for regular scale FDM, angled parts (the 3 -axis methods in this case) have higher strength than vertical ones [26], however it is unclear how this translates to single-walled prints.

The 5-axis tilt influences accuracy of tool endpoint, and this is shown in the slight decrease of accuracy and surface quality in beginning stages of print. This is compensated by preservation of quality at higher overhang angles. Still, in future work, it could be beneficial to restrict tilt even under the currently set tilt limit of $30^{\circ}$ degrees and use it only when beneficial. For example, if we take $15^{\circ}$ as the 
boundary under which multi-axis printing brings zero benefits, we should then exclude areas under $15^{\circ}$ overhang from tilt and use only necessary amount of tilt in areas over that limit.

\section{Conclusion}

Four different 3d printing methods were compared and the techniques required to perform them were presented. This includes the transformation based nonplanar vase mode slicing algorithm and the method of tilt limit for multi-axis robotic 3D printing along surface tangent. The goal of these methods was to preserve layer height and print path orientations at all overhang angles, increasing surface quality and strength. Mean deviation, surface roughness and layer adhesion strength were evaluated using 3D scanning and mechanical testing.

Some of the most important takeaways are the fact that using 5 -axis motion alone does not bring many noticeable improvements over the common 3-axis approach. And while nonplanar trajectories can bring improvements in strength and surface quality, the approach, if used on a conventional printing setup, is very limited in terms of printable geometry. Objects have to be either be divided into parts based on reach of the nozzle or 5-axis motion has to be used. Path planning for 5-axis motion brings another set of complications, in large part due to the need for collision checking.

But the combination of nonplanar trajectories and multi axis motion brings real expansion of capabilities for large scale FDM style printing. When printing large scale objects with large nozzle sizes, single line walls are often all that is necessary to achieve desired structural performance and this, combined with the limited ability of screw extruders to perform retractions, leads to the vase mode printing being a very suitable choice. Using this methods, stable wall thickness and large overhang angles of at least $75^{\circ}$ are achievable and quality of single walled objects is increased in regards to surface roughness at overhang angles over $15^{\circ}$ and mechanical strength, with $20 \%$ loss of maximum average delamination force for 5 -axis nonplanar trajectory at $45^{\circ}$ compared to $50 \%$ loss for 3 -axis planar trajectory.

\section{Declarations}

The Moai Head model was used under CC licence from: https://www.myminifactory.com/object/3dprint-moai-or-mo-ai-75141

Funding: This research was partially funded by a faculty project of FME BUT, FSI-S-20-6296.

Conflicts of interest/Competing interests: Not applicable

Availability of data and material: Included as a supplementary file.

Code availability: Included as a supplementary file.

Authors' contributions (optional: please review the submission guidelines from the journal whether statements are mandatory)

\section{Reference}

[1] T. D. Ngo, A. Kashani, G. Imbalzano, K. T. Q. Nguyen, and D. Hui, "Additive manufacturing (3D printing): A review of materials, methods, applications and challenges," Compos. Part B Eng., 2018.

[2] K. Thrimurthulu, P. M. Pandey, N. V. Reddy, and N. Venkata Reddy, "Optimum part deposition orientation in fused deposition modeling," International Journal of Machine Tools and

Manufacture, vol. 44, no. 6. pp. 585-594, 2004. 
[3] H. ming Zhao, Y. He, J. zhong Fu, and J. jiang Qiu, "Inclined layer printing for fused deposition modeling without assisted supporting structure," Robot. Comput. Integr. Manuf., vol. 51, no. May 2016, pp. 1-13, 2018.

[4] Y. Yang, J. Y. H. Fuh, H. T. Loh, and Y. S. Wong, "Multi-orientational deposition to minimize support in the layered manufacturing process," J. Manuf. Syst., vol. 22, no. 2, pp. 116-129, 2003.

[5] C. Gosselin, R. Duballet, P. Roux, N. Gaudillière, J. Dirrenberger, and P. Morel, "Large-scale 3D printing of ultra-high performance concrete - a new processing route for architects and builders," Mater. Des., vol. 100, pp. 102-109, Jun. 2016.

[6] H. Kalami and J. Urbanic, "Exploration of surface roughness measurement solutions for additive manufactured components built by multi-axis tool paths," Addit. Manuf., vol. 38, no. December 2020, p. 101822, 2021.

[7] Y. Pan, C. Zhou, Y. Chen, and J. Partanen, "Multitool and multi-axis computer numerically controlled accumulation for fabricating conformal features on curved surfaces," J. Manuf. Sci. Eng. Trans. ASME, vol. 136, no. 3, 2014.

[8] D. Bi, F. Xie, and K. Tang, "Generation of efficient iso-planar printing path for multi-axis fdm printing," J. Manuf. Mater. Process., vol. 5, no. 2, 2021.

[9] I. Mitropoulou, M. Bernhard, and B. Dillenburger, "Print Paths Key-framing: Design for nonplanar layered robotic FDM printing," Proc. - SCF 2020 ACM Symp. Comput. Fabr., no. December, 2020.

[10] J. R. Kubalak, A. L. Wicks, and C. B. Williams, "Exploring multi-axis material extrusion additive manufacturing for improving mechanical properties of printed parts," Rapid Prototyp. J., vol. 25, no. 2, pp. 356-362, 2019.

[11] D. Chakraborty, B. Aneesh Reddy, and A. Roy Choudhury, "Extruder path generation for Curved Layer Fused Deposition Modeling," CAD Comput. Aided Des., vol. 40, no. 2, pp. 235243, 2008.

[12] G. Zhao, G. Ma, J. Feng, and W. Xiao, "Nonplanar slicing and path generation methods for robotic additive manufacturing," Int. J. Adv. Manuf. Technol., vol. 96, no. 9-12, pp. 31493159, 2018.

[13] G. Schuh, G. Bergweiler, G. Lukas, S. Hohenstein, and J. Schenk, "Feature-based Print Method for Multi-Axis Material Extrusion in Additive Manufacturing," Procedia CIRP, vol. 93, pp. 8589, 2020.

[14] B. Ezair, S. Fuhrmann, and G. Elber, "Volumetric covering print-paths for additive manufacturing of 3D models," CAD Comput. Aided Des., vol. 100, pp. 1-13, 2018.

[15] R. J. A. Allen and R. S. Trask, "An experimental demonstration of effective Curved Layer Fused Filament Fabrication utilising a parallel deposition robot," Addit. Manuf., vol. 8, pp. 78-87, 2015.

[16] J. Etienne et al., "Curvislicer: Slightly curved slicing for 3-axis printers," ACM Trans. Graph., vol. 38, no. 4, 2019.

[17] N. R. Fry, R. C. Richardson, and J. H. Boyle, "Robotic additive manufacturing system for dynamic build orientations," Rapid Prototyp. J., vol. 26, no. 4, pp. 659-667, 2020.

[18] K. Xu, Y. Li, L. Chen, and K. Tang, "Curved layer based process planning for multi-axis volume printing of freeform parts," CAD Comput. Aided Des., vol. 114, pp. 51-63, 2019. 
[19] G. Fang, T. Zhang, S. Zhong, X. Chen, Z. Zhong, and C. C. L. Wang, "Reinforced FDM: Multi-axis filament alignment with controlled anisotropic strength," ACM Trans. Graph., vol. 39, no. 6, 2020.

[20] C. Dai, C. C. L. Wang, C. Wu, S. Lefebvre, G. Fang, and Y. J. Liu, "Support-free volume printing by multi-axis motion," ACM Trans. Graph., vol. 37, no. 4, 2018.

[21] S. Brell-Çokcan and J. Braumann, "Parametric Robot Control," Proc. 31st Annu. Conf. Assoc. Comput. Aided Des. Archit., pp. 242-251, 2011.

[22] A. Roschli et al., "Designing for Big Area Additive Manufacturing," Addit. Manuf., vol. 25, no. September 2018, pp. 275-285, 2019.

[23] S. Hertle, M. Drexler, and D. Drummer, "Additive Manufacturing of Poly(propylene) by Means of Melt Extrusion," Macromol. Mater. Eng., vol. 301, no. 12, pp. 1482-1493, 2016.

[24] C. E. Duty et al., "Structure and mechanical behavior of Big Area Additive Manufacturing (BAAM) materials," Rapid Prototyp. J., vol. 23, no. 1, pp. 181-189, 2017.

[25] G. Elber and E. Cohen, "Adaptive Isocurve-Based Rendering for Freeform Surfaces," ACM Trans. Graph., vol. 15, no. 3, pp. 249-263, 1996.

[26] J. R. C. Dizon, A. H. Espera, Q. Chen, and R. C. Advincula, "Mechanical characterization of 3Dprinted polymers," Addit. Manuf., vol. 20, pp. 44-67, 2018. 


\section{Supplementary Files}

This is a list of supplementary files associated with this preprint. Click to download.

- Collectedsuplementarydata.xlsx

- Nonplanarvaseslicer.png 